\title{
Regenerative treatment using a radioelectric asymmetric conveyor as a novel tool in antiaging medicine: an in vitro beta-galactosidase study
}

This article was published in the following Dove Press journal:

Clinical Interventions in Aging

27 June 2012

Number of times this article has been viewed

\author{
Salvatore Rinaldi ${ }^{1,2}$ \\ Margherita Maioli ${ }^{1,3,4}$ \\ Sara Santaniello $0^{3,4}$ \\ Alessandro Castagna ${ }^{1,2}$ \\ Gianfranco Pigliaru ${ }^{3,4}$ \\ Sara Gualini ${ }^{3,4}$ \\ Matteo Lotti Margotti ${ }^{5}$ \\ Arturo Carta ${ }^{6}$ \\ Vania Fontani ${ }^{1,2}$ \\ Carlo Ventura ${ }^{1,4,7}$ \\ 'Department of Regenerative \\ Medicine, Rinaldi Fontani Institute, \\ Florence; ${ }^{2}$ Department of Neuro \\ Psycho Physio Pathology and Neuro \\ Psycho Physical Optimization, \\ Rinaldi Fontani Institute, Florence; \\ ${ }^{3}$ Department of Biomedical Sciences, \\ University of Sassari, Sassari; \\ ${ }^{4}$ Laboratory of Molecular Biology \\ and Stem Cell Engineering, National \\ Institute of Biostructures and \\ Biosystems, Bologna; ${ }^{5}$ Department \\ of Information Technology and \\ Statistical Analysis, Rinaldi Fontani \\ Institute, Florence; ${ }^{6}$ Ophthalmology \\ Section, University of Parma, Parma; \\ ${ }^{7}$ Cardiovascular Department, \\ S Orsola Malpighi Hospital, University \\ of Bologna, Bologna, Italy
}

Correspondence: Salvatore Rinaldi Rinaldi Fontani Institute,

Viale Belfiore 43.

50144 Florence, Italy

Tel +390 55290307

Fax+39055290399

Email srinaldi@irf.it
Background: Beta-galactosidase is the most widely used biomarker for highlighting the processes of cellular aging, including neurodegeneration. On this basis, we decided to test in vitro whether a set of rescuing/reparative events previously observed by us in subjects treated with radioelectric asymmetric conveyor (REAC) technology may also involve antagonism of a marker of aging-related degenerative processes, as assessed by a reduction in beta-galactosidase at the cellular level.

Methods: Human adipose-derived stem cells were cultured at different passages, ranging from 5 to 20, with or without REAC exposure for 12 hours. The cells were then processed for biochemical beta-galactosidase staining and morphological microscopy analysis.

Results: We observed a significant reduction in expression of senescence associated-betagalactosidase, and a persistence of fibroblast-like morphology typical of human adipose-derived stem cells, even at late passages.

Conclusion: Our results indicate the ability of REAC technology to counteract in vitro senescence of human adipose-derived stem cells, and prompt the hypothesis that such technology may be exploited to antagonize in vivo senescence of tissue-resident or transplanted stem cells playing an important role in clinical treatment of age-related processes.

Keywords: aging, adipose-derived stem cells, neurodegenerative diseases

\section{Introduction}

Aging of the human population is a problem with many social and economic implications. If aging is accompanied by disease, especially of the neurodegenerative type, the social and economic costs will increase even more dramatically. The aging processes are natural phenomena, but are accelerated and aggravated by environmental factors, often beyond the control or knowledge of the subject. It is also very difficult to implement real and effective strategies that can slow down the processes of aging and related diseases in a "biological" manner. On the other hand, biomarkers are presently available for close monitoring of the aging process. One of the most often cited in the literature for highlighting both the processes of cellular aging and neurodegeneration is beta-galactosidase. ${ }^{1-4}$ We have previously shown that radioelectric asymmetric conveyer (REAC) technology, using specific protocols, is effective in eliciting reparative phenomena and is able to drive gene expression profiles controlling stem cell differentiation and pluripotency in vitro. Based on these findings, the purpose of this study was to verify whether REAC technology, using a specific protocol known as the "in vitro regenerative treatment protocol" (IVRTP), may be able to influence the in vitro production of beta-galactosidase from human adipose-derived stem cells 
which have been subjected to an aging process throughout multiple passages in culture.

\section{Materials and methods REAC technology for therapeutic use}

REAC is an innovative technology $y^{5,6}$ involving biostimulation and/or bioenhancement techniques that induce weak radioelectric currents in tissues, thereby inducing cell reprogramming activity. The model used in this study (ASMED, Florence, Italy) is specific for regenerative treatment. REAC technology has demonstrated efficacy in ameliorating several stress-related disorders, ${ }^{7-13}$ depression, ${ }^{12,14,15}$ anxiety, ${ }^{12,15}$ social anxiety, ${ }^{16}$ agoraphobia, ${ }^{17}$ bipolar disorder, ${ }^{18}$ behavioral and psychiatric symptoms in Alzheimer's disease, ${ }^{19}$ and impaired motor control. ${ }^{20-24}$ Recently, REAC technology using IVRTP has also demonstrated an ability to induce stem cell pluripotency and differentiation. ${ }^{25}$

\section{In vitro regenerative REAC protocol}

REAC IVRTP consists of a sequence of radiofrequency bursts $250 \mathrm{msec}$ in duration, with an off interval of 2.5 seconds. The REAC apparatus is placed into a $\mathrm{CO}_{2}$ incubator, set at a frequency of $2.4 \mathrm{GHz}$, and its conveyor electrodes are immersed into culture medium containing human adiposederived stem cells. The REAC-radiated power is about $2 \mathrm{~mW}$, the electric field is $0.4 \mathrm{~V} / \mathrm{m}$, the magnetic field is $1 \mathrm{~mA} / \mathrm{m}$, the specific absorption rate $0.128 \mu \mathrm{W} / \mathrm{g}$, and the density of radioelectric current flowing in the culture medium (J) during the REAC single radiofrequency burst is $30 \mu \mathrm{A} / \mathrm{cm}^{2}$.

\section{Isolation and culture of human adipose-derived stem cells}

According to the procedure approved by the local ethics committee, all tissue samples were obtained after informed consent. Human subcutaneous adipose tissue samples were obtained during lipoaspiration or liposuction procedures. After washing, the lipoaspirates were digested with $0.2 \%$ collagenase A type I solution (Sigma-Aldrich, St Louis, MO) under gentle agitation for 45 minutes at $37^{\circ} \mathrm{C}$, and centrifuged at $2000 \mathrm{rpm}$ for 10 minutes to separate the stromal vascular fraction from the adipocytes. If necessary, the mesenchymal stem cell fraction was treated with red blood cell lysis buffer for 5 minutes at $37^{\circ} \mathrm{C}$, then centrifuged again. The supernatant was discarded and the cell pellet was resuspended and seeded in culture flasks containing Dulbecco's modified Eagle's medium-low glucose supplemented with $20 \%$ heat-inactivated fetal bovine serum, $1 \%$ penicillinstreptomycin, and $2 \mathrm{mM} \mathrm{L-glutamine,} \mathrm{and} \mathrm{incubated} \mathrm{at}$ $37^{\circ} \mathrm{C}$ in a humidified atmosphere with $5 \% \mathrm{CO}_{2}$. When the cultures were near confluence, the cells were detached using trypsin, and seeded into 6-well tissue culture plates at the appropriate passages. The REAC apparatus ${ }^{5,6}$ was placed into a $\mathrm{CO}_{2}$ incubator, set at $2.4 \mathrm{GHz}$, and its conveyor electrodes were immersed for 12 hours in the culture medium containing human adipose-derived stem cells at passages $5,10,15$, and 20 .

\section{Senescence-associated beta-galactosidase staining}

Beta-galactosidase staining was performed using a senescence-associated $\beta$-galactosidase (SA- $\beta$-Gal) staining kit (Cell Signaling Technology, Danvers, MA) for 12 hours. Briefly, human adipose-derived stem cells cultured at passages 5, 10, 15, and 20 with or without REAC exposure were cultured in 6 -well plates $\left(3 \times 10^{3}\right.$ per well $)$ for 12 hours, fixed with fixative solution, and then processed according to the kit instructions. All the experiments were repeated three times, and one of the representative set of results is shown. The cells were then photographed under an inverted microscope at $100 \times$ magnification for qualitative detection of SA- $\beta$-Gal activity. The numbers of positive (blue) and negative cells were counted in five random fields under the microscope (at $200 \times$ magnification and bright field illumination), and the percentage of SA- $\beta$-Gal-positive cells was calculated as the number of positive cells divided by the total number of cells counted.

\section{Data analysis}

Statistical analysis of the data was performed using the Statistical Package for Social Science version 13 (SPSS Inc, Chicago, IL). For this study, nonparametric statistical tests were used, ie, the Kruskal-Wallis and Wilcoxon signedrank tests. $P<0.05$ was considered to be statistically significant.

\section{Results}

Figure 1 shows controls and REAC-treated human adipose-derived stem cells at passages 1, 5, 10, 15 and 20. Figure 2 shows beta-galactosidase staining in human adiposederived stem cells exposed or not exposed to REAC during the observation period of 50 days. It is evident that the number of SA- $\beta$-Gal-stained cells (blue) is already significantly reduced at passage 5 in human adipose-derived stem cells exposed to REAC, as compared with untreated cells at the same passage. This behavior becomes more evident with a progressive increment of this difference on the passage of 


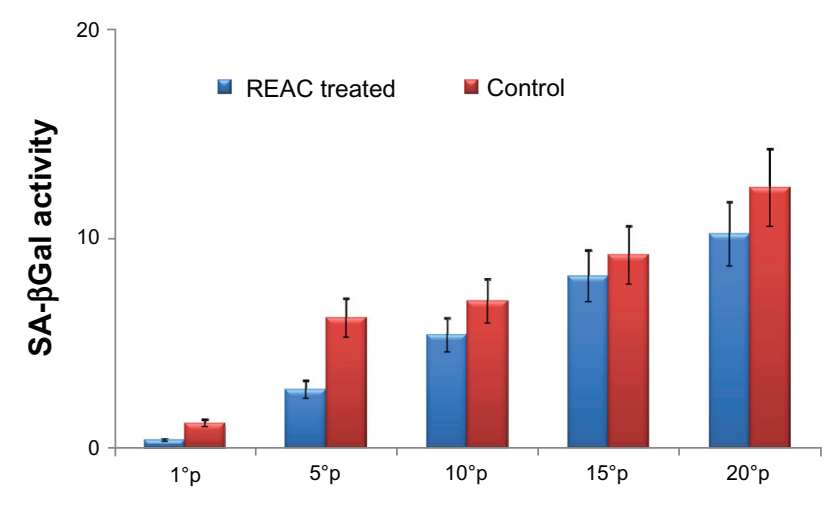

Figure I Effect of the radioelectric asymmetric conveyor on percentage of senescence-associated $\beta$-galactosidase-positive cells.

Notes: Human adipose-derived stem cells at passages I, 5, 10, 15, and 20 were cultured in 6-well plates at a concentration of $3 \times 10^{3}$ cells per well. Senescenceassociated $\beta$-galactosidase activity was measured after culturing for 12 hours with (blue line) or without the radioelectric asymmetric conveyor (red line) stimulus. The cells were then photographed under a microscope at $100 \times$ magnification for qualitative detection of senescence-associated $\beta$-galactosidase activity. The numbers of positive (blue) and negative cells were counted in five random fields under the microscope (at 200x magnification and bright field illumination), and the percentage of senescence-associated $\beta$-galactosidase-positive cells was calculated as the number of positive cells divided by the total number of cells counted. All the REAC-treated cells were significantly different from the untreated group (mean $\pm S D, n=3$, $P<0.05$ ).

Abbreviations: REAC, radioelectric asymmetric conveyor; SD, standard deviation.

time in culture. Moreover, in the last passages, in unexposed human adipose-derived stem cells both the number of SA- $\beta$ Gal-positive cells and the intensity of the staining were remarkably increased. In the same figures, it is evident that the cellular morphology differs between human adipose-derived

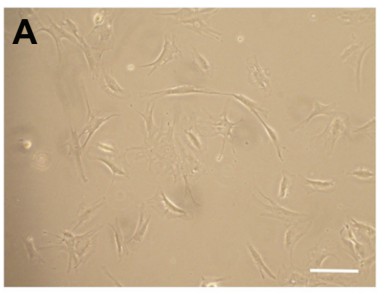

Control - time 0

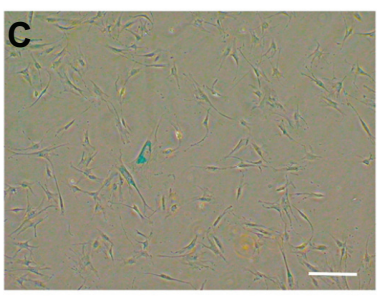

Control - passage 20

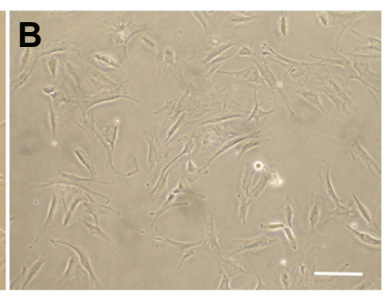

REAC treated - time 0

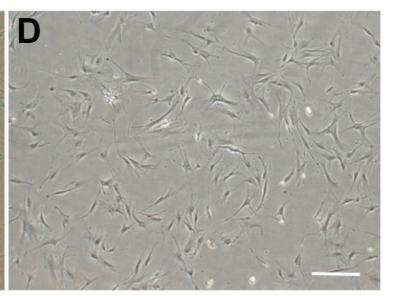

REAC treated - passage 20
Figure 2 Senescence-associated $\beta$-galactosidase activity.

Notes: Human adipose-derived stem cells at passages 0 (Panel $\mathbf{A}$ and $\mathbf{B}$ ) and 20 (Panel C and D) were plated into 6-well plates $\left(3 \times 10^{3}\right.$ cells per well). After overnight culture for attachment, the cells were cultured in the presence or absence of the radioelectric asymmetric conveyor for 12 hours. Human adipose-derived stem cells were then processed for senescence-associated $\beta$-galactosidase and photographed under a microscope at $100 \times$ magnification.

Abbreviation: REAC, radioelectric asymmetric conveyor. stem cells treated and not treated with REAC. In particular, unlike control cells, which developed a deranged morphology in their final passages, REAC-treated cells still showed their typical fibroblast-like morphology, even at passage 20.

\section{Discussion}

Aging is a natural part of life for any organism. At the cellular level, senescence tends to block cell proliferation irreversibly. The speed and quality with which this process occurs is determined by several factors, many of them determined at the environmental level and often with little or no chance of defense or protection. The lengthening of the average life span is a worldwide phenomenon, and it is now evident that this process may be associated with pathological aging, as in a number of neurodegenerative conditions, including Alzheimer's disease. Nowadays, there are still no effective therapies for such diseases, which result in considerable patient discomfort and impaired quality of life, as well as having a high socioeconomic impact. This scenario has prompted researchers to seek new strategies and tools for counteracting the onset and progression of aging at the cellular and molecular levels. Within this context, our results showing that REAC-IVRTP acts on human adipose-derived stem cells, a remarkable multipotent cell population, to counteract the onset of beta-galactosidase expression, which is one of the main biomarkers of aging and neurodegeneration, and may hold promise for future development in both cell therapy and antiaging. Such a perspective is reinforced by the already established clinical evidence for the efficacy of REAC technology, administered using specific protocols as a bioenhancer and neuroenhancer.

\section{Conclusion}

The findings of this study, albeit initial, open up the possibility of REAC technology being a cost-effective and easily applicable prevention tool in anti-aging medicine.

\section{Acknowledgment}

We would like to thank the Italian Society of Neuro Psycho Physical Optimization, which provided financial support for this study.

\section{Disclosure}

SR and VF are the inventors of the radioelectric asymmetric conveyor.

\section{References}

1. Geng YQ, Guan JT, Xu XH, Fu YC. Senescence-associated betagalactosidase activity expression in aging hippocampal neurons. Biochem Biophys Res Commun. 2010;396:866-869. 
2. Gruber HE, Ingram JA, Norton HJ, Hanley EN Jr. Senescence in cells of the aging and degenerating intervertebral disc: immunolocalization of senescence-associated beta-galactosidase in human and sand rat discs. Spine (Phila Pa 1976). 2007;32:321-327.

3. Yang NC, Hu ML. The limitations and validities of senescence associated-beta-galactosidase activity as an aging marker for human foreskin fibroblast Hs68 cells. Exp Gerontol. 2005;40:813-819.

4. Gerland LM, Peyrol S, Lallemand C, Branche R, Magaud JP, French M. Association of increased autophagic inclusions labeled for beta-galactosidase with fibroblastic aging. Exp Gerontol. 2003;38: 887-895.

5. Rinaldi S, Fontani V, assignees. Radioelectric asymmetric conveyor for therapeutic use. EP1301241. October 11, 2006.

6. Rinaldi S, Fontani V, assignees. Radioelectric asymmetric conveyor for therapeutic use. US Patent 7,333,859. February 19, 2008.

7. Collodel G, Moretti E, Fontani V, et al. Effect of emotional stress on sperm quality. Indian J Med Res. 2008;128:254-261.

8. Fontani V, Rinaldi S, Aravagli L, Mannu P, Castagna A, Margotti ML. Noninvasive radioelectric asymmetric brain stimulation in the treatment of stress-related pain and physical problems: psychometric evaluation in a randomized, single-blind placebo-controlled, naturalistic study. Int J Gen Med. 2011;4:681-686.

9. Rinaldi S, Fontani V, Aravagli L, Margotti ML. Psychological and symptomatic stress-related disorders with radio-electric treatment: psychometric evaluation. Stress Health. 2010;26:350-358.

10. Rinaldi S, Fontani V, Aravagli L, Mannu P. Psychometric evaluation of a radio electric auricular treatment for stress related disorders: a double-blinded, placebo-controlled controlled pilot study. Health Qual Life Outcomes. 2010;8:31.

11. Rinaldi S, Fontani V, Aravagli L, et al. Stress-related psychophysiological disorders: randomized single blind placebo controlled naturalistic study of psychometric evaluation using a radio electric asymmetric treatment. Health Qual Life Outcomes. 2011;9:54.

12. Rinaldi S, Fontani V, Moretti E, et al. A new approach on stress-related depression and anxiety: neuro-psycho- physical-optimization with radio electric asymmetric-conveyer. Indian J Med Res. 2010;132:189-194.

13. Fontani V, Aravagli L, Margotti ML, Castagna A, Mannu P, Rinald S. Neuropsychophysical optimization by REAC technology in the treatment of: sense of stress and confusion. Psychometric evaluation in a randomized, single blind, sham-controlled naturalistic study. Patient Prefer Adherence. 2012;6:195-199.

14. Mannu P, Rinaldi S, Fontani V, Castagna A, Margotti ML. Radio electric treatment vs es-citalopram in the treatment of panic disorders associated with major depression: an open-label, naturalistic study. Acupunct Electrother Res. 2009;34:135-149.
15. Olivieri EB, Vecchiato C, Ignaccolo N, et al. Radioelectric brain stimulation in the treatment of generalized anxiety disorder with comorbid major depression in a psychiatric hospital: a pilot study. Neuropsychiatr Dis Treat. 2011;7:449-455.

16. Fontani V, Mannu P, Castagna A, Rinaldi S. Social anxiety disorder: radio electric asymmetric conveyor brain stimulation versus sertraline. Patient Prefer Adherence. 2011;5:581-586.

17. Mannu P, Rinaldi S, Fontani V, Castagna A, Margotti ML. Noninvasive brain stimulation by radioelectric asymmetric conveyor in the treatment of agoraphobia: open-label, naturalistic study. Patient Prefer Adherence. 2011;5:575-580.

18. Mannu P, Rinaldi S, Fontani V, Castagna A. Long-term treatment of bipolar disorder with a radioelectric asymmetric conveyor. Neuropsychiatr Dis Treat. 2011;7:373-379.

19. Mannu P, Rinaldi S, Fontani V, Castagna A. Radio electric asymmetric brain stimulation in the treatment of behavioral and psychiatric symptoms in Alzheimer disease. Clin Interv Aging. 2011;6:207-211.

20. Castagna A, Rinaldi S, Fontani V, Mannu P. Radioelectric asymmetric brain stimulation and lingual apex repositioning in patients with atypical deglutition. J Multidiscip Healthc. 2011;4:209-213.

21. Castagna A, Rinaldi S, Fontani V, Aravagli L, Mannu P, Margotti ML. Does osteoarthritis of the knee also have a psychogenic component? Psycho-emotional treatment with a radio-electric device vs intraarticular injection of sodium hyaluronate: an open-label, naturalistic study. Acupunct Electrother Res. 2010;35:1-16.

22. Rinaldi S, Fontani V, Castagna A. Brain activity modification produced by a single radioelectric asymmetric brain stimulation pulse: a new tool for neuropsychiatric treatments. Preliminary fMRI study. Neuropsychiatr Dis Treat. 2011;7:649-654.

23. Fontani V, Rinaldi S, Castagna A, Margotti ML. Noninvasive radioelectric asymmetric conveyor brain stimulation treatment improves balance in individuals over 65 suffering from neurological diseases: pilot study. Ther Clin Risk Manag. 2012;8:73-78.

24. Mura M, Castagna A, Fontani V, Rinaldi S. Preliminary pilot fMRI study of neuropostural optimization with a noninvasive asymmetric radioelectric brain stimulation protocol in functional dysmetria. Neuropsychiatr Dis Treat. 2012;8:149-154.

25. Maioli M, Rinaldi S, Santaniello S, et al. Radio frequency energy loop primes cardiac, neuronal, and skeletal muscle differentiation in mouse embryonic stem cells: a new tool for improving tissue regeneration. Cell Transplant. September 22, 2011. [Epub ahead of print.]
Clinical Interventions in Aging

\section{Publish your work in this journal}

Clinical Interventions in Aging is an international, peer-reviewed journal focusing on evidence-based reports on the value or lack thereof of treatments intended to prevent or delay the onset of maladaptive correlates of aging in human beings. This journal is indexed on PubMed Central, MedLine, the American Chemical Society's 'Chemical Abstracts Ser-

\section{Dovepress}

vice' (CAS), Scopus and the Elsevier Bibliographic databases. The manuscript management system is completely online and includes a very quick and fair peer-review system, which is all easy to use. Visit http://www.dovepress.com/testimonials.php to read real quotes from published authors. 